\title{
Describing the population health burden of depression: health-adjusted life expectancy by depression status in Canada
}

\author{
C. Steensma, MSc (1,2); L. Loukine, MSc (1); H. Orpana, PhD (1,3); L. McRae, BSc (1); J. Vachon, MSc (1); \\ F. Mo, PhD (1); M. Boileau-Falardeau, MSc (1,2); C. Reid, MA (1); B. C. Choi, PhD (1,4,5)
}

This article has been peer reviewed.

Tweet this article

\begin{abstract}
Introduction: Few studies have evaluated the impact of depression in terms of losses to both premature mortality and health-related quality of life (HRQOL) on the overall population. Health-adjusted life expectancy (HALE) is a summary measure of population health that combines both morbidity and mortality into a single summary statistic that describes the current health status of a population.

Methods: We estimated HALE for the Canadian adult population according to depression status. National Population Health Survey (NPHS) participants 20 years and older ( $n=12$ 373) were followed for mortality outcomes from 1994 to 2009, based on depression status. Depression was defined as having likely experienced a major depressive episode in the previous year as measured by the Composite International Diagnostic Interview Short Form. Life expectancy was estimated by building period abridged life tables by sex and depression status using the relative risks of mortality from the NPHS and mortality data from the Canadian Chronic Disease Surveillance System (2007-2009). The Canadian Community Health Survey (2009/10) provided estimates of depression prevalence and Health Utilities Index as a measure of HRQOL. Using the combined mortality, depression prevalence and HRQOL estimates, HALE was estimated for the adult population according to depression status and by sex.
\end{abstract}

Results: For the population of women with a recent major depressive episode, HALE at 20 years of age was 42.0 years ( $95 \%$ CI: $40.2-43.8$ ) compared to 57.0 years ( $95 \%$ CI: $56.8-57.2$ ) for women without a recent major depressive episode. For the population of Canadian men, HALE at 20 was 39.0 years (95\% CI: 36.5-41.5) for those with a recent major depressive episode compared to 53.8 years (95\% CI: 53.6-54.0) for those without. For the 15.0-year difference in HALE between women with and without depression, 12.3 years can be attributed to the HRQOL gap and the remaining 2.7 years to the mortality gap. The 14.8 fewer years of HALE observed for men with depression equated to a 13.0-year HRQOL gap and a 1.8-year mortality gap.

Conclusion: The population of adult men and women with depression in Canada had substantially lower healthy life expectancy than those without depression. Much of this gap is explained by lower levels of HRQOL, but premature mortality also plays a role.

Keywords: life expectancy, healthy life expectancy, mortality, health-related quality of life, depression

\section{Introduction}

Depression contributes significantly to the burden of disease throughout the world, including in Canada. ${ }^{1}$ It is estimated that over 298 million people worldwide are living with depression. ${ }^{2}$ In 2012, about 3.2 million Canadians over the age of 15
Highlights

- Men and women in Canada who have depression live a substantially higher proportion of their life in an unhealthy state compared to their counterparts without depression.

- This gap in healthy life expectancy between Canadians with and without depression is primarily associated with losses in quality of life.

- Emotional state, cognitive state and pain are the key attributes associated with losses in quality of life for Canadians experiencing a recent major depressive episode.

- Based on observations from past studies of the Canadian household population, the burden of depression on healthy life expectancy at a population level appears to be greater than that associated with other chronic conditions such as diabetes, hypertension and obesity.

(11.3\%) reported having experienced symptoms consistent with a major depressive episode in their lifetime, while the prevalence of such an episode in the previous 12 months was $4.7 \%$ in this population. ${ }^{3}$ Women and young people aged 15 to 24 years experienced the highest prevalence of a 12-month major depressive episode. $^{3}$

Depression has an important impact on health-related quality of life (HRQOL), functioning, mortality due to intentional injury and health care utilization. ${ }^{4-6}$ In

Author references:

1. Health Promotion and Chronic Disease Prevention Branch, Public Health Agency of Canada, Government of Canada, Ottawa, Ontario, Canada

2. Department of Social and Preventive Medicine, Université de Montréal, Montréal, Quebec, Canada

3. School of Psychology, University of Ottawa, Ottawa, Ontario, Canada

4. Dalla Lana School of Public Health, University of Toronto, Toronto, Ontario, Canada

5. Injury Prevention Research Center, Shantou University Medical College, Shantou, China

Correspondence: Bernard Choi, Health Promotion and Chronic Disease Prevention Branch, Public Health Agency of Canada, 785 Carling Avenue, Ottawa, ON K1A 0K9; Tel: 613-797-3821; Fax: 613-941-2057; Email: Bernard.Choi@phac-aspc.gc.ca 
addition to these direct negative outcomes, depression has also been demonstrated to increase risk for coronary heart disease, ${ }^{7}$ stroke $^{8}$ and cancer ${ }^{9}$ and a decline in physical functioning. ${ }^{10}$ Potential mechanisms for the relationship between depression and physical disease include immune and endocrine dysregulation and inflammatory processes. ${ }^{11}$ Depression has also been associated with an increased risk of mortality in general community populations, as well as in patient populations with chronic illnesses such as coronary heart disease, cancer, diabetes and stroke. ${ }^{11}$ There are likely reciprocal effects between depression and disease, with depression being a risk factor for, and a sequela of, disease. ${ }^{11}$

Summary measures of population health using the Global Burden of Disease methodology $^{1,12-15}$ have ranked depression very high on the list of health conditions contributing to the global and national burden of disease, particularly in terms of losses due to disability. Major depressive episode is the second leading cause of years lived with disability globally ${ }^{1}$ as well as in the United States, ${ }^{12}$ the United Kingdom, ${ }^{13}$ China $^{14}$ and Canada. ${ }^{15}$

Less well documented is the association of depression with life expectancy and healthy (or disease-free) life expectancy. Understanding both life expectancy and healthy life expectancy among people who have depression will help to better characterize its disease burden. A recent systematic review and meta-analysis ${ }^{16}$ concluded that people living with a mood disorder have a mortality rate twice as high as those without a mood disorder, and potential years of life lost due to mental disorders ranged from 1.4 to 32 years, with a median of 10.1 years. Jia et al. ${ }^{17}$ reported that adults living with depression in the United States experienced a 28.9 year loss of quality adjusted life expectancy (QALE) at age 18 compared to those without depression. Results from the few existing studies on healthy or disease-free life expectancy and depression are not consistent. ${ }^{18-20}$ A number of these studies are restricted to older adults and thus cannot be generalized to the entire population. These studies also use a measure of functional health restricted to activities of daily living, which do not consider attributes such as pain, emotion and cognition.
The objective of our study was to estimate period life expectancy (LE) and healthadjusted life expectancy (HALE) of Canadian adults (aged 20 years and older) according to depression status. Note that the period approach to estimating LE and HALE adopted in this paper is a summary measure of population health for a given period. Period life expectancy estimates the hypothetical life expectancy of an individual were they to experience the ageand sex-specific mortality rates in a given period. This should not be confused with projected life expectancy based on modelling or cohort life expectancy based on the actual mortality experience of a specific cohort. In a similar fashion, period HALE is a hypothetical estimate reflecting an individual's healthy life expectancy were they to experience the age- and sexspecific mortality and age- and sexspecific HRQOL levels at a given point in time.

These estimates are useful to better understand the population health impact of a condition. They are also useful for informing policy and programs, and for making decisions about the relative burden of specific health conditions. Because of the varied course of depression, with both chronic and episodic cases included in the population studied, the estimates in this study should not be applied to predict the expected health course of any individual.

\section{Methods}

\section{Data sources}

To estimate HALE, several types of data are necessary: all-cause mortality rates by depression status, depression prevalence and HRQOL estimates by depression status. All-cause mortality rates for the Canadian adult population with and without depression were estimated based on a methodology that partitions rates for total population into mortality rates by disease categories using a mortality relative risk or hazard ratios and a prevalence of those categories. The methodology is described in detail in our previous study. ${ }^{21}$

We used data from the following three sources:

- National Population Health Survey (NPHS), for estimating mortality hazard ratios (HRs) by depression status;
- Canadian Community Health Survey (CCHS), for estimating depression prevalence and HRQOL by depression status; and

- Canadian Chronic Disease Surveillance System (CCDSS), for actual age- and sex-specific all-cause mortality rates in the Canadian population, which were then partitioned into those associated with depression and those not associated with depression, based on the mortality HR estimated using the NPHS and depression prevalence estimated from CCHS.

The NPHS is a longitudinal survey conducted by Statistics Canada of 17276 Canadians of all ages living in households in the 10 provinces. The NPHS has a biennial follow-up spanning the years $1994 / 95$ to $2010 / 11$ and includes death clearance against the Canadian Mortality Database. ${ }^{22}$ These data were used to estimate mortality HR associated with depression required for estimating all-cause mortality rates for people with and without depression. Our study population at baseline included 12373 participants aged 20 years and older.

The CCHS is an annual cross-sectional survey, conducted by Statistics Canada, of a sample of approximately 65000 Canadians aged 12 years and older living in households in the provinces and territories. ${ }^{23}$ Our study used a two-year CCHS sample (2009-2010) and included 103815 participants aged 20 years and older. We used these data to estimate the prevalence of depression, as well as to attribute depression status in estimating all-cause mortality rates (see "Analysis" section for more detail). We also obtained Health Utilities Index (HUI) scores by depression status from the CCHS. We estimated depression prevalence and HUI scores from the CCHS because the data were more recent, and the larger sample size allowed for more accurate estimation of depression and HUI scores.

We used CCDSS all-cause mortality data for the period of 2007-2009 in the study. The CCDSS collects administrative data that include death and population counts by sex and five-year age groups for all residents of all ages in all provinces and territories, who use the public health care systems. Both mortality and population size information come from provincial and territorial health insurance databases 
that cover about $97 \%$ of the Canadian population. Data are collected from all Canadian provinces and territories and summarized by the Public Health Agency of Canada. ${ }^{24}$

\section{Measures}

Depression is measured in the CCHS and NPHS using the Composite International Diagnostic Interview Short Form (CIDI-SF) instrument. The CIDI is a structured diagnostic interview, based on diagnostic criteria from the International Classification of Diseases (ICD-10) and the Diagnostic and Statistical Manual of Mental Disorders (DSM-IV), that is administered by trained interviewers. The short form of the Interview is based on a subset of CIDI questions that could still reliably reproduce prevalence estimates. ${ }^{25}$ The CIDI-SF interview produces scores that give predicted probabilities of depression. For this study, respondents with a predicted probability of 0.9 and above were considered to have experienced a major depressive episode during the previous year. The CIDI-SF was optional content on the 2009-2010 CCHS, and not all provinces and territories chose to include this module. Estimates of depression are based on partial provincial and territorial coverage that includes five provinces (Prince Edward Island, Quebec, Saskatchewan, Alberta and British Columbia) and two territories (Nunavut and Northwest Territories).

HRQOL is measured using the Health Utilities Index Mark 3 instrument in the CCHS. The HUI is a preference-based measure of HRQOL based on responses to questions about functioning for the following eight attributes: vision, hearing, speech, ambulation, dexterity, emotion, cognition and pain. ${ }^{26}$ Single-attribute utility scores range from 0.0 (lowest level of functioning) to 1.0 (full functional capacity). The eight attributes are combined into an overall score that ranges from 1.00 (perfect health) through 0.00 (death) to -0.36 (the worst possible health state; from a preference perspective, some health states are considered worse than death and are consequently assigned negative scores). A change of 0.03 or more in overall HUI scores and 0.05 or more in single-attribute utility scores is considered clinically important. ${ }^{27}$

The HUI has been widely used, and its validity and reliability in a variety of applications is supported..$^{27,28}$ A study assessing the sensitivity to depression outcomes of several multiattribute utility indexes found HUI to be able to discriminate well between levels of severity in the two depression instruments being evaluated. ${ }^{29}$ The HUI score is used as a morbidity measure in the estimation of HALE. Depression typically has a negative impact on the emotional state, including sustained negative affect and difficulties experiencing positive affect. ${ }^{30}$ In order to assess whether HRQOL differences are due exclusively to changes in the HUI emotion attribute, we compared HUI scores (all ages combined) by depression status for each of the eight attributes.

\section{Analysis}

Relative risks of mortality by depression status for women and for men were approximated by HRs. The HRs were estimated by fitting sex-specific discrete-time proportional models with a complementary log-log function using the completed NPHS data. We defined people with depression as those with a high probability (0.9) of having had a major depressive episode in the 12 months prior to data collection in at least one NPHS cycle, according to a method described by Simpson et al. ${ }^{31}$ The first episode defined a case date. Respondents were followed up for mortality events every two years and a variable for each cycle was included in the model (cycle 1-cycle 9) as a time-interval measure. The sex-specific models were adjusted by age. As the HRs were used to estimate only all-cause mortality rates associated with depression, we did not adjust the models for any other comorbidities, socioeconomic status or other determinants of health. Because of the complex sample design of this survey, we used the bootstrapping method to calculate variance and produce $95 \%$ confidence intervals (CIs) for HR. ${ }^{22}$ Age- and sex-specific depression prevalences, required for decomposing total mortality rates by depression status, were estimated using the CCHS 2009-2010. Mean HUI estimates by age, sex and depression status were also calculated. Bootstrapping was used to generate $95 \% \mathrm{CI}^{23}$ In our study, total mortality rates by sex and 5-year age groups were estimated from CCDSS data for the period of 2007-2009. In this study, ageand sex-specific mortality rates for people with depression and for those without depression were estimated by decomposing mortality rates for the total population following the methodology we described in our previous study. ${ }^{21}$

We used the Chiang method ${ }^{32}$ to generate period (2007-2009) sex-specific abridged life tables by depression using 14 standard age groups $(20-24,25-29, \ldots, 80-84, \geq 85$ years). The Gompertz function was used to provide an accurate estimate of LE for the last open-ended 85-years-plus age interval in order to close the life table, as described by Hsieh. ${ }^{33}$ The modified Sullivan method ${ }^{34}$ was used for HALE estimation. According to this method the "life-years lived" was adjusted by the HUI.

$$
L_{x}^{\prime}=L_{x} * H U I_{x}
$$

where $L_{x}^{\prime}$ is adjusted life-years lived in age-interval $x, L_{x}$ is life-years lived in age-interval $x$ and $H U I_{x}$ is Health Utilities Index Mark 3 for people in age-interval $x$.

The variance of LE and HALE was estimated using bootstrap methodology. Statistics Canada's surveys provide 500 bootstrap weights for variance estimation to account for complex survey designs. ${ }^{22,23}$ Using those weights, 500 sets of HR estimates from NPHS and 500 sets of prevalence of depression and HUI estimates from CCHS were generated and all unique combinations of those estimates used to obtain mortality rates to build 250000 life tables by depression and sex. This allowed estimating LE and HALE variance, building CIs around point estimates and conducting $\mathrm{z}$ tests to determine the statistical significance of the differences in LE and HALE. The $95 \%$ CIs were built based on the normality assumption. Due to the nature of the study population (adults 20 years and older), LE and HALE results were estimated at age 20 years and not birth.

The Arriaga decomposition, or partitioning, method ${ }^{35}$ (adapted for the Sullivan method $^{34}$ ) was applied to quantify which part of HALE differences according to depression status can be attributable to differences in premature mortality and which are attributable to loss of HRQOL (morbidity). For each age group, the change in HALE between the comparison groups is partitioned into the following components:

$\triangle H A L E=\triangle M O R B+\triangle M O R T=\frac{L_{x 1}+L_{x 2}}{2} \Delta H U I+\frac{H U I_{1}+H U I_{2}}{2} \Delta L_{x}$ 
where $\triangle M O R B$ estimates change due to HRQOL, $\triangle M O R T$ estimates change due to mortality, $L_{x 1}$ and $H U I_{x 1}$ refer to the number of years lived and HUI score respectively for those with depression for age-interval $x$, and $L_{x 2}$ and $H U I_{x 2}$ refer to the number of years lived and HUI score respectively for those without depression for age-interval $x$. (More details about how this methodology is applied to healthy life expectancy estimations can be found elsewhere. ${ }^{21,36}$ )

\section{Results} istics of the participants in the NPHS and CCHS, the two national health surveys that we used for this study. The prevalence of major depressive episode in our study population $(2009 / 10)$ was $5.5 \%$ $(6.7 \%$ for women and $4.2 \%$ for men; results not shown).
Table 1 shows the demographic character-

Based on analysis of the NPHS data, there were 2154 deaths over the 16-year followup period. Mortality risk was significantly higher for those who experienced a major depressive episode (age-adjusted HR = 1.43; $95 \%$ CI: $1.22-1.68)$. This significant risk persisted when we restricted analyses to women only (age-adjusted HR = 1.55; 95\% CI: 1.28-1.87), while the risk for men was nonsignificant (age-adjusted $\mathrm{HR}=1.28$; $95 \% \mathrm{CI}: 0.98-1.68$ ) (data not shown).

Unadjusted HRQOL values (as measured by HUI scores) varied by age, sex and depression status (see Table 2). HUI scores were considerably lower in all age groups for men and women who had experienced a major depressive episode during the preceding 12 months compared to those who had not experienced such an episode. According to definitions of disability categories based on global HUI scores developed by Feng et al., ${ }^{37}$ men and women

TABLE 1

Demographic characteristics of survey participants, NPHS 1994/95 and CCHS 2009/10

\begin{tabular}{|c|c|c|}
\hline Characteristics & NPHS 1994/95 & CCHS 2009/10 \\
\hline Age, mean (range) in years & $45(20-100)$ & $47.5(20-102)$ \\
\hline \multicolumn{3}{|l|}{ Sex, ${ }^{a} \%(95 \% \mathrm{Cl})$} \\
\hline Male & $48.6(48.3-48.9)$ & $49.1(49.1-49.1)$ \\
\hline Female & $51.4(51.1-51.7)$ & $50.9(50.9-50.9)$ \\
\hline \multicolumn{3}{|l|}{ Marital status, ${ }^{\mathrm{b}} \%(95 \% \mathrm{Cl})$} \\
\hline Married/common-law & $68.3(67.3-69.3)$ & $65.7(65.1-66.3)$ \\
\hline Single/widowed/divorced/separated & $31.7(30.7-32.7)$ & $34.3(33.7-34.9)$ \\
\hline \multicolumn{3}{|l|}{ Highest level of education, ${ }^{\mathrm{c}} \%(95 \% \mathrm{Cl})$} \\
\hline Less than high school & $25.7(24.5-26.8)$ & $14.4(14.1-14.8)$ \\
\hline High school graduation & $40.7(39.5-41.9)$ & $23.7(23.3-24.2)$ \\
\hline Post-secondary graduation & $33.6(32.5-34.8)$ & $61.8(61.3-62.4)$ \\
\hline \multicolumn{3}{|l|}{ Depression, ${ }^{\mathrm{d}} \%(95 \% \mathrm{Cl})$} \\
\hline Yes & NA & $5.5(5.2-5.9)$ \\
\hline No & NA & $94.5(94.1-94.8)$ \\
\hline $\begin{array}{l}\text { Depression }{ }^{d} \text { in at least one cycle (1994-2008), } \\
\%(95 \% \mathrm{Cl})\end{array}$ & & - \\
\hline Yes & $4.7(4.4-4.9)$ & NA \\
\hline No & $95.3(95.0-95.6)$ & NA \\
\hline
\end{tabular}

Abbreviations: CCHS, Canadian Community Health Survey; CIDI-SF, Composite International Diagnostic Interview Short Form; NA, not applicable; NPHS, National Population Health Survey.

a $\mathrm{n}=12373$ (NPHS); $\mathrm{n}=103815$ (CCHS).

${ }^{\mathrm{b}} \mathrm{n}=12371$ (NPHS); $\mathrm{n}=103636$ (CCHS).

' $n=12347$ (NPHS); $n=101783$ (CCHS).

${ }^{\mathrm{d}}$ Based on responses to CIDI-SF, indicative of having experienced a major depressive disorder in the previous year. $\mathrm{n}=3501$ (NPHS); $\mathrm{n}=48355$ (CCHS). with depression experienced on average moderate disability (HUI < 0.89) at all age groups, whereas only older $(\geq 55$ years) men and women without depression fell into this category. Similarly, men with depression on average experienced severe disability (HUI $<0.70$ ) at age 40 years while women experienced this at age 45 years; average HUI scores for men and women without depression did not drop below this threshold at any age group in our study.

An assessment of each of the eight HUI attributes by sex (all ages combined) showed that depression was associated with a clinically meaningful lower score (i.e. a difference of 0.05 or higher) for the emotion, pain and cognition attributes (Table 2).

Both LE and HALE for women with depression were lower than for those without depression; LE for men with depression was not significantly lower whereas HALE was (Table 3). LE at age 20 was 4.1 years (95\% CI: 1.1-7.1) lower for women with depression compared to those without, whereas HALE at age 20 was 15.0 years (95\% CI: $13.2-16.8$ ) lower for women with depression. For men, the gap in LE at age 20 between those with and without depression was 2.7 years (95\% CI: 0.0-5.4), whereas HALE at age 20 for men with depression was 14.8 years (95\% CI: 12.3-17.4) lower. LE and HALE at age 65 were lower for both women and men with depression. Women with depression had an LE at age 65 years that was 3.2 years (95\% CI: $1.8-4.6$ ) lower and a HALE at age 65 that was 6.7 years $(95 \%$ CI: 5.3-8.1) lower than women without depression. LE at age 65 years for men with depression was 2.1 years $(95 \% \mathrm{CI}$ : 0.1-4.1) lower than that of men without depression. HALE at age 65 years was 6.0 years (95\% CI: 3.8-8.2) less for men with depression compared to those without depression.

An assessment of the individual contribution of loss of HRQOL and premature mortality to differences in HALE at age 20 indicated that, for the 15.0-year difference in HALE between women with and without depression, 12.3 years could be attributed to HRQOL losses and the remaining 2.7 years to mortality losses. The 14.8 fewer years of HALE for men with depression equated to a 13.0-year HRQOL gap 
TABLE 2

Health-related quality of life status by sex, age, HUI attribute and depression status ${ }^{\mathrm{a}}$, Canada, 2009/10

\begin{tabular}{|c|c|c|c|c|}
\hline & \multicolumn{4}{|c|}{ Average Health Utilities Index score $(95 \% \mathrm{CI})$} \\
\hline & $\begin{array}{c}\text { Women without } \\
\text { depression }\end{array}$ & Women with depression & Men without depression & Men with depression \\
\hline \multicolumn{5}{|c|}{ Age group (years) } \\
\hline $20-24$ & $0.93(0.92-0.94)$ & $0.82(0.77-0.87)$ & $0.93(0.92-0.94)$ & $0.74(0.65-0.83)$ \\
\hline $25-29$ & $0.94(0.93-0.95)$ & $0.81(0.76-0.85)$ & $0.93(0.92-0.94)$ & $0.81(0.76-0.87)$ \\
\hline $30-34$ & $0.93(0.93-0.94)$ & $0.74(0.65-0.84)$ & $0.93(0.92-0.94)$ & $0.78(0.71-0.85)$ \\
\hline $35-39$ & $0.93(0.92-0.94)$ & $0.74(0.64-0.84)$ & $0.93(0.92-0.94)$ & $0.80(0.74-0.87)$ \\
\hline $40-44$ & $0.91(0.90-0.92)$ & $0.73(0.68-0.78)$ & $0.91(0.89-0.92)$ & $0.68(0.60-0.77)$ \\
\hline $45-49$ & $0.91(0.89-0.92)$ & $0.63(0.54-0.71)$ & $0.92(0.90-0.93)$ & $0.67(0.58-0.76)$ \\
\hline $50-54$ & $0.90(0.89-0.91)$ & $0.69(0.63-0.75)$ & $0.91(0.90-0.91)$ & $0.66(0.59-0.73)$ \\
\hline $55-59$ & $0.88(0.87-0.89)$ & $0.71(0.65-0.77)$ & $0.88(0.87-0.89)$ & $0.59(0.49-0.70)$ \\
\hline $60-64$ & $0.88(0.87-0.89)$ & $0.68(0.62-0.75)$ & $0.90(0.89-0.91)$ & $0.62(0.52-0.72)$ \\
\hline $65-69$ & $0.87(0.86-0.88)$ & $0.59(0.49-0.69)$ & $0.88(0.87-0.89)$ & $0.60(0.49-0.71)$ \\
\hline $70-74$ & $0.86(0.85-0.88)$ & $0.67(0.58-0.77)$ & $0.87(0.85-0.88)$ & $0.63(0.45-0.81)$ \\
\hline $75-79$ & $0.81(0.78-0.83)$ & $0.66(0.54-0.78)$ & $0.83(0.81-0.85)$ & $0.69(0.39-1.00)$ \\
\hline $80-84$ & $0.79(0.77-0.82)$ & $0.48(0.16-0.81)$ & $0.79(0.76-0.82)$ & $0.36(-0.01-0.73)$ \\
\hline$\geq 85$ & $0.72(0.69-0.75)$ & $0.52(0.37-0.67)$ & $0.74(0.70-0.78)$ & $0.44(0.10-0.79)$ \\
\hline \multicolumn{5}{|l|}{ HUI attribute } \\
\hline Vision & $0.99(0.99-0.99)$ & $0.99(0.98-0.99)$ & $0.99(0.99-0.99)$ & $0.99(0.99-0.99)$ \\
\hline Speech & $1.00(1.00-1.00)$ & $1.00(1.00-1.00)$ & $1.00(1.00-1.00)$ & $1.00(1.00-1.00)$ \\
\hline Pain & $0.97(0.97-0.97)$ & $0.92^{\mathrm{b}}(0.91-0.93)$ & $0.98(0.97-0.98)$ & $0.93^{\mathrm{b}}(0.91-0.94)$ \\
\hline Mobility & $0.99(0.99-0.99)$ & $0.99(0.98-0.99)$ & $1.00(1.00-1.00)$ & $0.99(0.98-0.99)$ \\
\hline Hearing & $1.00(1.00-1.00)$ & $1.00(1.00-1.00)$ & $1.00(1.00-1.00)$ & $0.99(0.99-1.00)$ \\
\hline Emotion & $0.99(0.99-0.99)$ & $0.94^{\mathrm{b}}(0.93-0.95)$ & $0.99(0.99-0.99)$ & $0.92^{\mathrm{b}}(0.91-0.93)$ \\
\hline Dexterity & $1.00(1.00-1.00)$ & $1.00(1.00-1.00)$ & $1.00(1.00-1.00)$ & $1.00(1.00-1.00)$ \\
\hline Cognition & $0.98(0.98-0.98)$ & $0.93^{\mathrm{b}}(0.92-0.94)$ & $0.98(0.98-0.98)$ & $0.93^{\mathrm{b}}(0.92-0.94)$ \\
\hline
\end{tabular}

Abbreviations: Cl, confidence interval; CIDI-SF, Composite International Diagnostic Interview Short Form; HUI, Health Utilities Index.

Note: Light shading signifies moderate disability (global HUI score of $0.70-0.88$ ); dark shading signifies severe disability (global HUI score $<0.70$ ); no shading represents either no disability (global HUI score $=1.00$ ) or mild disability (global HUI score $=0.89-0.99$ ).

${ }^{a}$ Based on responses to CIDI-SF, indicative of having experienced a major depressive episode in the previous year.

${ }^{\text {b } C l i n i c a l l y ~ m e a n i n g f u l ~ d i f f e r e n c e ~ i n ~ a t t r i b u t e-s p e c i f i c ~ H U I ~ s c o r e ~ b e t w e e n ~ t h o s e ~ w i t h ~ a n d ~ w i t h o u t ~ d e p r e s s i o n . ~}$

and a 1.8-year mortality gap (data not shown).

We found large differences between adult Canadians with and without depression in terms of the percentage of their life spent in an unhealthy state (calculated as [LEHALE]/LE; see Figure 1). Both men and women with depression spent almost three times as much of their life expectancy at age 20 in poor health when compared to those without depression $(31 \%$ vs. $12 \%$ for females and $32 \%$ vs. $11 \%$ for males). These large differences persisted across age groups: based on LE and HALE at age 65 , men and women with depression were still living approximately twice as long in poor health as men and women of the same age without depression (40\% vs. $19 \%$ for women and $43 \%$ vs. $17 \%$ for men).

\section{Discussion}

In this study, we found significantly lower LE at age 20 for women, and HALE at age 20 for both women and men, among Canadians reporting symptoms consistent with a major depressive episode in the previous 12 months. We found this across age groups, although gaps in the proportion of life expectancy spent in an unhealthy state were greater among men and younger age groups with depression.
Although direct comparisons with other health problems need to be interpreted with caution, we found that losses of HALE associated with depression in the Canadian adult population were larger than those observed for obesity class 2 and above, ${ }^{21}$ and for diabetes and hypertension $^{38}$ in the same population. In addition, while those studies found a greater loss of HALE among women than men, we demonstrated approximately equal losses for both sexes.

Our results align with those of Jia et al., ${ }^{17}$ who reported a 28.9-year QALE loss at age 18 for adults with depression, which is substantively larger than the approximately 
TABLE 3

Life expectancy and HALE at age 20-24 and at 65-69, by sex and depression status ${ }^{\mathrm{a}}$, Canada, 2009/10

\begin{tabular}{lccccc} 
& \multicolumn{4}{c}{ Years $(95 \% \mathrm{CI})$} \\
\hline & $\begin{array}{c}\text { Age group, } \\
\text { years }\end{array}$ & $\begin{array}{c}\text { Women } \\
\text { without } \\
\text { depression }\end{array}$ & $\begin{array}{c}\text { Women with } \\
\text { depression }\end{array}$ & $\begin{array}{c}\text { Men without } \\
\text { depression }\end{array}$ & $\begin{array}{c}\text { Men with } \\
\text { depression }\end{array}$ \\
\hline $\begin{array}{l}\text { Life } \\
\text { expectancy }\end{array}$ & $20-24$ & $\begin{array}{c}64.9 \\
(64.8-65.0)\end{array}$ & $\begin{array}{c}60.8 \\
(59.0-62.6)\end{array}$ & $\begin{array}{c}60.4 \\
(60.3-60.5)\end{array}$ & $\begin{array}{c}57.7 \\
(55.0-60.4)\end{array}$ \\
\cline { 2 - 6 } & $65-69$ & 22.4 & 19.2 & 19.1 & 17.0 \\
\hline $\begin{array}{l}\text { Health- } \\
\text { adjusted life } \\
\text { expectancy }\end{array}$ & $20-24$ & $(22.3-22.5)$ & $(17.8-20.6)$ & $(19.1-19.1)$ & $(15.0-19.0)$ \\
\cline { 2 - 6 } & $65-69$ & 57.0 & 42.0 & 53.8 & 39.0 \\
& $(56.8-57.2)$ & $(40.2-43.8)$ & $(53.6-54.0)$ & $(36.5-41.5)$ \\
\hline
\end{tabular}

Abbreviations: $\mathrm{Cl}$, confidence interval; CIDI-SF, Composite International Diagnostic Interview Short Form; HALE, healthadjusted life expectancy.

${ }^{a}$ Based on responses to CIDI-SF, indicative of having experienced a major depressive episode in the previous year.

15-year loss of HALE at age 20 for adults with depression that we report. This may be explained both by real differences in the health experience of the Canadian and US populations and by methodological differences between our studies. Moreover, the hazard ratio for mortality associated with depression we observed $(\mathrm{HR}=1.43)$ is somewhat smaller than the relative risk of mortality of 2.08 for adults

FIGURE 1

Percentage of life spent in an unhealthy state ${ }^{a}$, by sex, age and depression status ${ }^{b}$, Canada, 2009/10

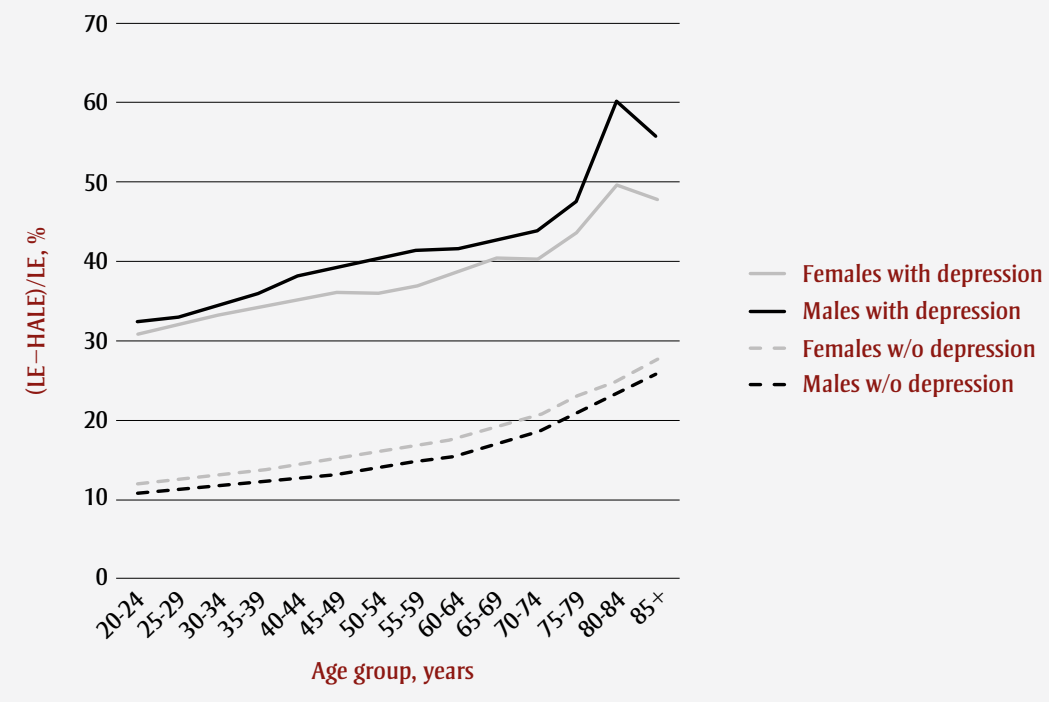

Abbreviations: CIDI-SF, Composite International Diagnostic Interview Short Form; HALE, health-adjusted life expectancy; LE, life expectancy; w/o, without.

a (LE-HALE)/LE.

${ }^{\mathrm{b}}$ Based on responses to CIDI-SF, indicative of having experienced a major depressive episode in the previous year. chronic diseases was reduced by approximately three years. While these studies found decreases in health expectancy in their participants living with depression, the magnitude of these decreases is less than we found in our study, except for male participants in the Reynolds et al. ${ }^{19}$ study. The larger differences may be due to the fact that our measure of HRQOL included attributes not found in the Activities-of-DailyLiving measure of disability used in those three studies.

We found that a large portion of the lower HALE in participants with depression was due to lower levels in HRQOL. A comparison of each HUI attribute (Table 2) demonstrated that, although there is a clinically meaningful difference between men and women with and without depression for the emotion attribute, there are also meaningful differences for the pain and cognition attributes. Although the association between pain and depression is well documented, the relationship is complex and causal pathways are not thoroughly understood: data support both a model where depression leads to changes in the individual that increase their vulnerability to pain, as well as scenarios where pain symptoms are a risk factor for future depression. ${ }^{39}$ Cognitive dysfunction has also been found in a large proportion of patients with depression and has been demonstrated to exist early on in the course of depression; it may even precede diagnosis. ${ }^{40}$

In addition to the expected lower values of HALE associated with lower HRQOL, we also found that a considerable amount of the decrease could be attributed to premature mortality. While our results found women demonstrated the largest losses of life expectancy, other studies on mortality risk and life expectancy according to depression status found that men had the greater mortality risk or loss of life expectancy. ${ }^{11,17,19,20}$ However, most of these studies tended to focus on elderly or older adult populations that likely have a different risk profile than the full adult population. Indeed, Shah et al. ${ }^{41}$ assessed both sex and age differences in the association of depression with mortality and found significant depression-age-sex interactions: mortality risk increased for men as age increased above 55 years while the inverse was found for women. Further study should be undertaken in non-elderly populations in order to better understand this phenomenon. 


\section{Strengths and limitations}

Our study benefitted from comprehensive data used to estimate LE and HALE across the age spectrum of adults in Canada. The survey data we used are from large, population-based samples of the Canadian household population: the NPHS allowed us to follow the mortality experience of over 12000 adult Canadians for 16 years, a longer period than any of the other studies evaluating the association of depression status with life expectancy and/or healthy life expectancy.

Our study has several limitations. When estimating mortality risk, we only considered the first observed episode of probable depression based on the CIDI-SF and did not include depression status at subsequent follow-up. This could have led to misclassification of subjects whose depression status changed.

The definition of depression used in this study (predicted probability of major depressive episode of 0.9 ) is consistent with the recommended use of the CIDI-SF instrument and corresponds to reporting five to nine symptoms consistent with depression, including one of two cardinal symptoms. This measure was developed for the National Comorbidity Survey in the United States. A 0.9 predicted probability is a high threshold that likely results in more false negatives than false positives, and thus will underestimate, rather than overestimate the burden of depression in Canada. ${ }^{42}$

The CIDI-SF is an optional item in the CCHS and, as such, does not include responses from all Canadian provinces and territories, which may limit the representativeness of our results. We assessed the impact of the missing jurisdictions using an earlier CCHS cycle $(2000 / 01)$ that included major depressive episode results for all provinces and territories. Age- and sex-specific major depressive episode prevalences from this cycle did not change appreciably when we removed the jurisdictions missing from the 2009/10 cycle, suggesting that representativeness of our study population was not affected by the missing jurisdictions. It should also be pointed out that our measure of depression, recent major depressive episode, does not adequately capture losses in healthy life expectancy specific to longerterm, chronic depression.
The CCHS is a household survey, and by excluding other populations, such as those living in institutions and long-term care facilities, it is possible that the prevalence of depression does not reflect that of the entire Canadian population. There may be differential non-response on the NPHS and CCHS: people with depression may be less likely to respond, resulting in an underestimation of the prevalence of depression. However, this would mean that our estimates are conservative, and that the true burden of depression may be higher than we report.

Our study aimed to describe the association between depression and healthy life expectancy and did not seek to understand the modifying influence of socioeconomic status or of other health conditions. However, in describing the mortality and morbidity of people with depression, it would be inappropriate to adjust for comorbid conditions. The influence on healthy life expectancy of health conditions that are comorbid with depression is unclear. While Pérès et al. ${ }^{20}$ only found significant differences in healthy life expectancy between those with and without depression among those reporting three or more chronic conditions, Reynolds et al. ${ }^{19}$ found large, significant differences in healthy life expectancy when comparing those with depression to those without in the absence of chronic diseases. Further study is needed to determine the impact of these risk factors and other potentially positive modifying effects, such as social participation, on healthy life expectancy. ${ }^{18}$

Finally, the approach to summarizing population health in this study represents the life and healthy life expectancy experience by a population at a given point in time, based on age- and sex-specific mortality and HRQOL estimates. These period estimates of life expectancy and HALE should only be interpreted as summary measures of population health, and not as the life and healthy life expectancies of any real individual.

\section{Conclusion}

This study demonstrates that, at the Canadian population level, women who have recently experienced a major depressive episode have a significantly lower period life expectancy and HALE at age 20 years than those who have not; for men, period HALE at age 20 is significantly lower for those who recently experienced a major depressive episode. Losses in HALE due to lower HRQOL are considerable and, while not as large, losses due to increased mortality risk also contribute to this difference, particularly among women. These findings demonstrate a high burden of depression in the Canadian population.

\section{Acknowledgements}

This research received no specific grant from any funding agency in the public, commercial or not-for-profit sectors. There were no competing interests.

Colin Steensma contributed to the study design, developed the first draft of the article, and critically reviewed the article. Lidia Loukine contributed to the study design, conducted all statistical analyses, wrote parts of the article, and critically reviewed the article. Heather Orpana contributed to the study design, wrote parts of the article, and critically reviewed the article. Louise McRae contributed to the study design and critically reviewed the article. Julie Vachon contributed to the study analysis and data interpretation and critically reviewed the article. Frank Mo, Michèle Boileau-Falardeau and Carrie Reid contributed to the data interpretation and critically reviewed the article. Bernard Choi contributed to the study concept and critically reviewed the article.

Data used in this study were accessed through sharing agreements with Statistics Canada and the Canadian provinces and territories. Other researchers can access Statistics Canada data through the Data Liberation Initiative of Statistics Canada.

\section{References}

1. Ferrari AJ, Charlson FJ, Norman RE, et al. Burden of depressive disorders by country, sex, age, and year: findings from the Global Burden of Disease Study 2010. PLoS Med. 2013;10(11):e1001547. doi: 10.1371 /journal.pmed.1001547.

2. Ferrari AJ, Charlson FJ, Norman RE, et al. The epidemiological modelling of major depressive disorder: application for the Global Burden of Disease Study 2010. PloS One. 2013;8(7): e69637. doi: 10.1371/journal.pone .0069637 . 
3. Pearson C, Janz T, Ali J. Mental and substance use disorders in Canada. Ottawa (ON): Statistics Canada; 2013.

4. Ruo B, Rumsfeld JS, Hlatky MA, Liu $\mathrm{H}$, Browner WS, Whooley MA. Depressive symptoms and healthrelated quality of life: the Heart and Soul Study. JAMA. 2003;290(2): 215-21. doi: 10.1001/jama.290.2.215.

5. Rihmer Z. Suicide risk in mood disorders. Curr Opin Psychiatry 2007 Jan;20(1):17-22. doi: 10.1097/yco .0b013e3280106868.

6. Stephens T, Joubert N. The economic burden of mental health problems in Canada. Chronic Dis Can. 2001; 22(1):18-23. doi: 10.1017/gmh.2014.2.

7. Brown $\mathrm{AD}$, Barton DA, Lambert GW. Cardiovascular abnormalities in patients with major depressive disorder. CNS Drugs. 2009;23(7):583-602. doi: 10.2165/00023210-200923070-00004.

8. Pan A, Sun Q, Okereke OI, Rexrode $\mathrm{KM}, \mathrm{Hu}$ FB. Depression and risk of stroke morbidity and mortality: a meta-analysis and systematic review. JAMA. 2011;306(11):1241-9. doi: 10.1001/jama.2011.1282.

9. Currier MB, Nemeroff CB. Depression as a risk factor for cancer: from pathophysiological advances to treatment implications. Annu Rev Med. 2014;65:203-21. doi: 10.1146/annurev -med-061212-171507.

10. Penninx BW, Guralnik JM, Ferrucci L, Simonsick EM, Deeg DJ, Wallace RB. Depressive symptoms and physical decline in community-dwelling older persons. JAMA. 1998;279(21):1720-6. doi: 10.1001/jama.279.21.1720.

11. Cuijpers P, Vogelzangs N, Twisk J, Kleiboer A, Li J, Penninx BW. Is excess mortality higher in depressed men than in depressed women? A meta-analytic comparison. J Affect Disord. 2014;161: 47-54. doi: 10.1016/j.jad.2014.03.003.

12. Murray CJ, Abraham J, Ali MK, et al. The state of US health, 1990-2010: burden of diseases, injuries, and risk factors. JAMA. 2013;310(6):591-606. doi: 10.1001/jama.2013.13805.

13. Murray CJ, Richards MA, Newton JN, et al. UK health performance: findings of the Global Burden of Disease Study 2010. Lancet. 2013;381(9871): 997-1020. doi: 10.1016/ s0140-6736(13)60355-4.
14. Yang G, Wang Y, Zeng Y, et al. Rapid health transition in China, 1990-2010: findings from the Global Burden of Disease Study 2010. Lancet. 2013; 381(9882):1987-2015. doi: 10.1016/ s0140-6736(13)61097-1.

15. Ratnasingham S, Cairney J, Manson $\mathrm{H}$, Rehm J, Lin E, Kurdyak P. The burden of mental illness and addiction in Ontario. Can J Psychiatry 2013;58(9):529-37. doi: 10.1002/wps .20321 .

16. Walker ER, McGee RE, Druss BG Mortality in mental disorders and global disease burden implications: a systematic review and meta-analysis. JAMA Psychiatry. 2015;72(4):334-41. doi: 10 .1001/jamapsychiatry.2014.2502.

17. Jia H, Zack MM, Thompson WW, Crosby AE, Gottesman II. Impact of depression on quality-adjusted life expectancy (QALE) directly as well as indirectly through suicide. Soc Psychiatry Psychiatr Epidemiol. 2015; 50(6):939-49. doi: 10.1007/s00127-015 -1019-0.

18. Chiao C, Lee S, Liao W, et al. Social participation and life expectancythe case of older adults in Taiwan from 1996 to 2003. Int J Gerontol. 2013;7(2):97-101. doi: 10.1016/j.ijge .2012.07.001.

19. Reynolds SL, Haley WE, Kozlenko N. The impact of depressive symptoms and chronic diseases on active life expectancy in older Americans. Am J Geriatr Psychiatry. 2008;16(5):425-32 doi: 10.1097/jgp.0b013e31816ff32e.

20. Pérès $\mathrm{K}$, Jagger $\mathrm{C}$, Matthews $\mathrm{FE}$. Impact of late-life self-reported emotional problems on Disability-Free Life Expectancy: results from the MRC Cognitive Function and Ageing Study. Int J Geriatr Psych. 2008; 23(6):643-9. doi: 10.1002/gps.1955.

21. Steensma C, Loukine L, Orpana H, Lo E, Choi B, Waters C, et al. Comparing life expectancy and health-adjusted life expectancy by body mass index category in adult Canadians: a descriptive study. Popul Health Metr. 2013;11(1):21. doi: 10.1186/1478-7954 $-11-21$.
22. Tambay JL, Catlin G. Sample design of the National Population Health Survey. Health Rep. 1995;7(1):29-38.

23. Béland Y. Canadian Community Health Survey - methodological overview. Health Reports. 2002;13(3):9-14.

24. Dai S, Robitaille C, Bancej C, Loukine L, Waters C, Baclic O. Executive summary: report from the Canadian Chronic Disease Surveillance System: hypertension in Canada, 2010. Chronic Dis Can. 2010;31(1):46-7.

25. Kessler RC, Barker PR, Colpe LJ, et al. Screening for serious mental illness in the general population. Arch Gen Psychiatry. 2003;60(2):184-9.

26. Feeny D, Furlong W, Torrance GW, et al. Multiattribute and single-attribute utility functions for the Health Utilities Index Mark 3 system. Med Care. 2002;40(2):113-28.

27. Horsman J, Furlong W, Feeny D, Torrance G. The Health Utilities Index (HUI): concepts, measurement properties and applications. Health Qual Life Outcomes. 2003;1:54.

28. Feeny D, Huguet N, McFarland BH, Kaplan MS. The construct validity of the Health Utilities Index Mark 3 in assessing mental health in population health surveys. Qual Life Res. 2009;18(4):519-26.

29. Mihalopoulos C, Chen G, Iezzi A, Khan MA, Richardson J. Assessing outcomes for cost-utility analysis in depression: comparison of five multiattribute utility instruments with two depression-specific outcome measures. Br J Psychiatry. 2014;205(5): 390-7. doi: 10.1192/bjp.bp.113.136036.

30. Joormann J, Quinn ME. Cognitive processes and emotion regulation in depression. Depress Anxiety. 2014; 31 (4):308-15. doi: 10.1002/da.22264.

31. Simpson KR, Meadows GN, Frances AJ, Patten SB. Is mental health in the Canadian population changing over time? Can J Psychiatry 2012;57(5): 324-31.

32. Chiang CL. The life table and its applications. Malabar (FL): Krieger; 1984. 
33. Hsieh JJ. A general theory of life table construction and a precise abridged life table method. Biom J. 1991;33(2): 143-62.

34. Sullivan DF. A single index of mortality and morbidity. HSMHA Health Rep. 1971;86(4):347-54.

35. Arriaga EE. Measuring and explaining the change in life expectancies. Demography. 1984;21(1):83-96.

36. Nusselder WJ, Looman CW. Decomposition of differences in health expectancy by cause. Demography. 2004;41(2):315-34

37. Feng Y, Bernier J, McIntosh C, Orpana $\mathrm{H}$. Validation of disability categories derived from Health Utilities Index Mark 3 scores. Health Rep. 2009; 20(2):43-50.
38. Public Health Agency of Canada Steering Committee on Health-Adjusted Life Expectancy. Health-adjusted life expectancy in Canada: 2012 report by Public Health Agency of Canada. Ottawa (ON): Public Health Agency of Canada; 2012. Available from: http:// healthycanadians.gc.ca/publications /science-research-sciences-recherches /health-adjusted-life-expectancy -canada-2012-esperance-vie-ajusteefonction-etat-sante/index-eng.php

39. Goesling J, Clauw DJ, Hassett AL. Pain and depression: an integrative review of neurobiological and psychological factors. Curr Psychiatry Rep. 2013;15(12):1-8. doi: 10.1007 /s11920-013-0421-0.
40. Trivedi MH, Greer TL. Cognitive dysfunction in unipolar depression: implications for treatment. J Affect Disord. 2014;152:19-27. doi: 10.1016/j .jad.2013.09.012.

41. Shah AJ, Ghasemzadeh N, ZaragozaMacias E, Patel R, Eapen DJ, Neeland $\mathrm{IJ}$, et al. Sex and age differences in the association of depression with obstructive coronary artery disease and adverse cardiovascular events. J Am Heart Assoc. 2014;3(3):e000741. doi: 10.1161/JAHA.113.000741.

42. Patten SB, Brandon-Christie J, Devji J, Sedmak B. Performance of the Composite International Diagnostic Interview Short Form for major depression in a community sample. Chronic Dis Can. 2000;21(2):68-72.

\section{Call for Submissions}

\section{Health Promotion and Chronic Disease Prevention in Canada}

\begin{abstract}
The Health Promotion and Chronic Disease Prevention in Canada Editors are pleased to announce that the journal once again welcomes articles from all authors, regardless of affiliation.
\end{abstract}

For more information and to submit a manuscript please visit: phac-aspc.gc.ca/publicat/hpcdp-pspmc/authinfo-eng.php\#sub

\section{Research, Policy and Practice}

